\title{
Chronic kidney disease-mineral bone disease (CKD- MBD) and cardiac arrest risk factors among renal failure patients
}

\begin{abstract}
The major complications related to CKD include cardiovascular disease, anemia, and abnormalities related to mineral bone metabolism. Disturbance in mineral and bone metabolism accompanied by soft tissue and vascular calcification is one of the most common and important consequences of CKD development and progression. Sudden cardiac arrest is the leading cause of death for patients with end stage renal disease) ESRD (. The potential factors increasing the risk for sudden cardiac arrest in patients with CKD include fluid overload, abnormalities in ion metabolism, and electrolyte shifts. Hyperkalemia is associated with increases in the all-cause mortality and advanced CKD is an independent predictor of hyperkalemia. This study aimed to investigate the prevalence of cardiac arrest and CKD-MBD risk factors, as electrolyte imbalance, defect in mineral metabolism, anemia, and diabetes among CKD patients. This study was performed in El-Zahraa hospital in the West of Libya and conducted on 72 patients ( 35 males and 37 females) with chronic renal failure from January to June 2013 and a group of 40 (20 males and 20 females) individuals as control. By estimating GFR, most of patients (about $80 \%$ ) were in stage five of chronic kidney disease. The patients who within stage four limits were about $17 \%$ is very low percentage of patients who within stage three limits (about 3\%), on the other hand, no patients within limits of stages one or two. By measuring serum electrolytes for patients and control groups, the results showed that about half of the patient groups were with hyponatremia and hyperkalemia. Calcium levels in patients was lower than control in about $40 \%$, and Phosphorus level was higher than normal in $85 \%$ of patient populations. The illustrated results were showed that more than $50 \%$ of patients had increase in the alkaline phosphatase enzyme activity. The results also illustrated that most of patients were anemic $(80 \%)$ and fifty percent of patients were diabetic.
\end{abstract}

Keywords: bone disease, cardiac arrest, electrolytes, risk factors, etiology, anemia, hyperkalemia, dispersion, bone volume, bone turnover, bone mineralization, creatinine, sodium, potassium, calcium
Volume 3 Issue 5 - 2017

\author{
Azab Elsayed Azab,' Ata Sedik Ibrahim \\ Elsayed $^{2}$ \\ 'Department of Zoology, Zawia University, Libya \\ ${ }^{2}$ Department of Biomedical Sciences, Dar Al Uloom University, \\ Saudi Arabia
}

\begin{abstract}
Correspondence: Azab Elsayed Azab, Department of Zoology, Faculty of Science, Alejelat, Zawia University, Libya,
\end{abstract} Email azabelsaied@yahoo.com

Received: June 16,2017 | Published: August 16, 2017

\section{Introduction}

Chronic kidney disease (CKD) is defined as a disease characterized by alterations in either kidney structure or function or both for a minimum of 3 months duration. It can be caused by a variety of conditions and this introduces significant heterogeneity in terms of how patients with CKD may present to their clinicians. However, irrespective of the etiology of CKD, patients with mild CKD are mostly asymptomatic and symptoms are generally related to $\mathrm{CKD}$ complications that are observed in the late stages. The major complications related to CKD include cardiovascular disease, anemia, infectious complications, neuropathy and abnormalities related to mineral bone metabolism. Disturbance in mineral and bone metabolism accompanied by soft tissue and vascular calcification is one of the most common and important consequences of CKD development and progression. ${ }^{1}$ CKD is characterized by increased cardiovascular risk partly due to increased prevalence of traditional cardiovascular risk factors such as hypertension and diabetes mellitus and also due to direct increased cardiovascular risk from nontraditional cardiovascular risk factors such as anemia, malnutrition inflammation syndrome, retention of uremic toxins and chronic kidney disease-mineral bone disease (CKD-MBD). ${ }^{2} \mathrm{CKD}$ patients are also at high risk to develop end stage renal disease (ESRD) and risks for all-cause as well as cardiovascular mortality is even higher for ESRD compared with CKD patients. ${ }^{3}$

Sudden cardiac arrest is the leading cause of death for patients with ESRD. The potential factors increasing the risk for sudden cardiac arrest in patients with CKD include fluid overload, cardiomyopathy, ischemic heart disease, corrected QT interval dispersion, sympathetic over activity, obstructive sleep apnea, baroreflex ineffectiveness, inflammation, iron overload, abnormalities in ion metabolism, and electrolyte shifts. ${ }^{4}$ Jain et al. ${ }^{5}$ reported that hyperkalemia is associated with increases in the all-cause mortality and advanced CKD is an independent predictor of hyperkalemia. Sudden cardiac arrest is the most common cause of death for ESKD patients treated with hemodialysis, occurring at a rate 30 times greater than the general population. ${ }^{6}$ Even if a hemodialysis patient is able to be resuscitated after experiencing SCA, the likelihood of long-term survival is exceedingly low. The study of Bleyer et al..$^{7}$ showed that a reduction in glomerular filtration rate alone enhances sudden cardiac arrest risk, but it is evident that exposure to conventional hemodialysis augments this risk even further. Certainly, a higher prevalence of traditional cardiac risk factors such as advanced age, ischemic cardiomyopathy, diabetes, and preexisting arrhythmic heart disease is observed among 
patients with CKD, but whether these factors predict sudden cardiac arrest during a hemodialysis treatment remains unclear. ${ }^{6}$

In addition to effects on mortality and progression to ESRD, CKD is marked by disturbance in mineral and bone metabolism accompanied by soft tissue and vascular calcification which is one of the most common and important consequences of CKD development and progression. This systemic disorder is now referred to as CKDmineral and bone disorder (CKD-MBD). These alterations include biochemical abnormalities in calcium, phosphorous, parathyroid hormone (PTH), vitamin D, fibroblast growth factor-23 (FGF-23), changes in bone morphology such as bone volume, bone turnover, bone mineralization and calcification of soft tissue and blood vessels. ${ }^{3}$ This study aimed to investigate the prevalence of cardiac arrest and CKD-MBD risk factors, as electrolyte imbalance, defect in mineral metabolism, anemia, and diabetes among CKD patients.

\section{Materials and methods}

This study was conducted on 72 patients ( 35 males and 37 females) with chronic renal failure from January to June 2013 and a group of 40 (20 males and 20 females) individuals as control. Ethical approve and patients consent statement were taken from everyone and the study was performed in El-Zahraa hospital in the west of Libya. At first, all patients with proven chronic renal failure were included in study. In order to eliminate effects of sex and age on comparison between cases and control groups, age and sex were selected in each pair of groups as similar as possible. Demographic and anthropometric data including age, sex, weight, height, BMI and blood pressure were measured for the participants. All patients and normal participants were Libyans, above 18 years of age, and free from chronic degenerative diseases such as cancer or peritonitis. Five $\mathrm{mL}$ of blood was drawn by venous puncture. Collected blood sample was emptied in plain vial for biochemical tests. After clotting of blood in the plain vial, serum was separated, within an hour; by centrifugation at 3000-5000g for $5 \mathrm{~min}$. Serum was used for measurements of urea, creatinine, Sodium, Potassium, Calcium, Phosphorous and glucose levels. Laboratory standard operation procedures were maintained for all laboratory analysis. Internal quality control sera, both normal and pathological, were also run for each lot of the test, for the validation of the results. Biochemical studies were performed using commercially available kits from Biomeriux (France) according to the manufacturer's instructions. Sodium and potassium ions were determined using $\mathrm{Na}+$ and $\mathrm{K}+$ filter of flame photometer (Jenway PFP7. ESSEX.UK.) Defining variables CKD was defined as reduced excretory function with an estimated GFR (eGFR) $<60 \mathrm{~mL} / \mathrm{min} / 1.73 \mathrm{~m}^{2}$ as a marker of kidney dysfunction.

Furthermore, CKD was defined and classified into five stages of CKD as per National Kidney Foundation guidelines. The formula of Cockcroft and Gault equation (8) was used to calculate eGFR.

$\mathrm{eGFR}=[140$-age (in years) $]$ xweight (in $\mathrm{kg}$ )/[72xserum creatinine $(\mathrm{mg} / \mathrm{dl})]$

\section{Statistical analysis}

Data of patients and control groups were entered in electronic form using Microsoft Excel 2010.

The data were analyzed and presented using descriptive statistics (absolute and relative numbers, measures of means and standard deviation) and appropriate statistical tests ( $\chi 2 /$ test, Mann-Whitney $\mathrm{U}$ test, Fisher test, correlation, and frequency tests), Kolmogorov-
Smirnova and Shapiro-Wilk tests were used to see that data is following normal distribution or not. Those parameters which were not following normal distribution were analyzed by nonparametric tests using Graphpad prism analytical software. A probability level or $\mathrm{p}$ value less than $0.05(\mathrm{P}<0.05)$ was considered statistically significant.

\section{Results}

The patients of this study are 35 males and 37 females were diagnosed as chronic kidney disease patients by physical and laboratory investigations. By estimating GFR, most of patients were in stage five of chronic kidney disease, as illustrated in Figure 1, 80\% of males and $75.6 \%$ of female were within this stage. The patients who within stage four limits are $17.1 \%$ for males and $18.9 \%$ for females, but there is very low percentage of patients who within stage three limits $(2.9 \%$ for males and 5.5 for females), on the other hand, no patients within limits of stages one or two. The tabulated data in Table 1 , showed the significant differences of electrolytes levels between patient and control groups. These illustrated data in Table 1 and Figure 2 , showed decrease in mean serum sodium concentration by $-4.3 \%$ for males and $-5 \%$ for females compared to corresponding control groups, $57.1 \%$ of male patients and $56.7 \%$ of female patients were showed decrease in serum sodium ion concentration, this decrease did not show correlation with renal function tests as illustrated in Table 2. On the other hand, Potassium levels were showed correlation with eGFR, Creatinine concentrations and blood urea. The illustrated data in Figure 3 and Table 1, showed that about $43 \%$ of patients in both genders were hyperkalemic and $57 \%$ were normal, the percentage of mean differences of increase were $32.4 \%$ and $27.7 \%$ for male and female patients respectively.

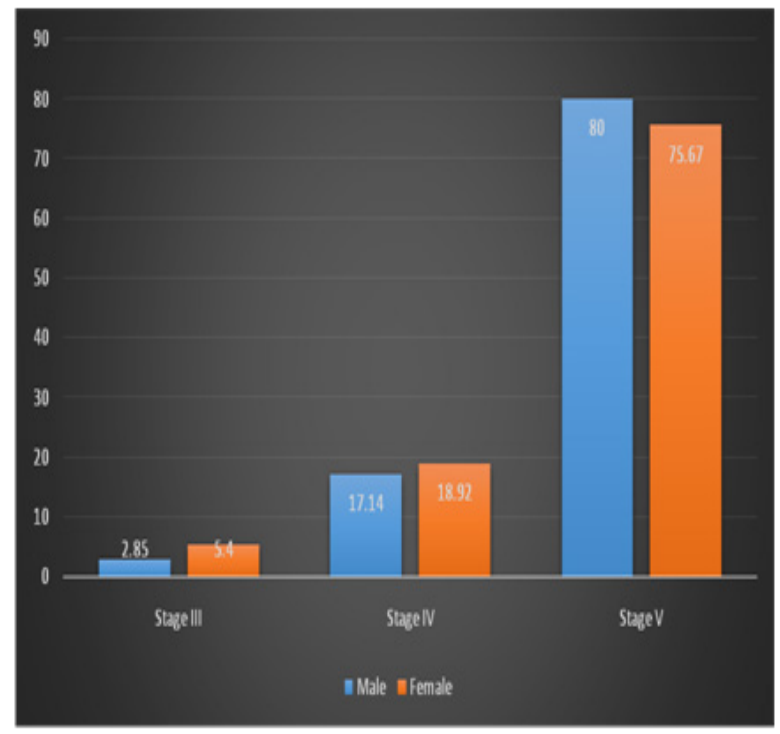

Figure I Distribution of patient groups according to stage of CKD.

Calcium levels in serum were illustrated in Figure 4 and Table 1 which showed decrease in serum calcium in both genders by $-10.4 \%$ and $-11.8 \%$ for male and female patients respectively. The data also illustrate that $42.8 \%$ of male and $37.8 \%$ of female patients showed decrease in Calcium levels in serum, the correlation tests in Table 2, did not show any correlation between Calcium levels and renal function tests. On the other hand, Phosphorus concentrations in plasma were showed correlation with blood urea as indicator of kidney function. The illustrated data in Figure 5, showed that, about $85 \%$ of patients were hyper phosphatemic and only $15 \%$ were normal, 
the percentage of mean differences between patients and control were $75.7 \%$ and $63.3 \%$ and Calcium Phosphorus product also showed

Table I Biochemical parameters in CKD patients and control increase in patient groups by $63.6 \%$ and $47 \%$ in male and female patients respectively.

\begin{tabular}{|c|c|c|c|c|c|c|c|c|c|}
\hline Gender & Groups & $\begin{array}{l}\mathrm{Na}^{+} \\
(\mathrm{mEq} / \mathrm{L}) \\
\mathrm{M} \pm \mathrm{SD}\end{array}$ & $\begin{array}{l}\mathrm{K}^{+} \\
(\mathrm{mEq} / \mathrm{L}) \\
\mathrm{M} \pm \mathrm{SD}\end{array}$ & $\begin{array}{l}\mathrm{Ca}^{++}(\mathrm{mg} / \\
\mathrm{dl}) \mathrm{M} \pm \mathrm{SD}\end{array}$ & 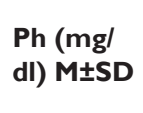 & 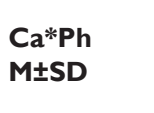 & $\begin{array}{l}\text { ALP } \\
\text { (IU/L) } \\
M \pm S D\end{array}$ & $\mathrm{Hb}(\mathrm{g} / \mathrm{dl})$ & $\begin{array}{l}\text { FBS }(\mathrm{mg} / \\
\text { dl) }\end{array}$ \\
\hline \multirow{2}{*}{ Males } & Control & $140 \pm 2.29$ & $4.16 \pm 0.29$ & $9.3 \pm 0.55$ & $\begin{array}{l}3.67 \pm \\
0.479\end{array}$ & $33.5 \pm 4.5$ & $94.9 \pm 17.1$ & $13.7 \pm 0.99$ & $89.6 \pm 12.4$ \\
\hline & Patients & $134 * * \pm 6.0 \mid$ & $5.51 * * \pm 1.07$ & $8.33 * \pm 1.29$ & $6.45 * * \pm 2.6$ & $54.8^{* *} \pm 16.8$ & $198 * * \pm 109$ & $10.8^{* * \pm 1.79}$ & $|5| * * \pm 86.6$ \\
\hline \multirow{2}{*}{ Females } & Control & $140 * * \pm 2.13$ & $4.15 \pm 0.34$ & $9.43 \pm 0.479$ & $3.65 \pm 0.47$ & $34.4 \pm 4.6$ & $93.5 \pm 19.5$ & $12.6 \pm 0.74$ & $94 \pm 11.6$ \\
\hline & Patients & $133 \pm 5.87$ & $5.3 * * \pm 1.3$ & $8.32 * \pm 1.7$ & $5.96 * * \pm 1.7$ & $50.6 * * \pm 13.8$ & $153 * * \pm 57$ & $9.74 * * \pm 1.74$ & $163 * * \pm 94.3$ \\
\hline
\end{tabular}

(*) significant difference compared to control group $(\mathrm{P}<0.05)$.

$(* *)$ highly significant difference compared to control group $(\mathrm{P}<0.0 \mathrm{I})$.

Table 2 Correlation between serum electrolytes and kidney function tests

\begin{tabular}{|c|c|c|c|c|c|}
\hline \multirow{5}{*}{$\begin{array}{l}\text { Correlation between eGFR } \\
\text { and Electrolytes }\end{array}$} & \multirow{2}{*}{ Spearman $r$} & $\mathrm{Na}^{+}$ & $\mathbf{K}^{+}$ & $\mathrm{Ca}^{++}$ & $\mathbf{P h}$ \\
\hline & & -0.213 & -0.385 & 0.0599 & -0.043 \\
\hline & $95 \%$ confidence interval & $\begin{array}{l}-0.518 \text { to } \\
0.140\end{array}$ & $\begin{array}{l}-0.643 \text { to } \\
-0.0489\end{array}$ & $\begin{array}{l}-0.305 \text { to } \\
0.409\end{array}$ & -0.380 to 0.304 \\
\hline & $P$ value (two-tailed) & 0.2194 & 0.0225 & 0.7446 & 0.806 \\
\hline & $P$ value summary & ns & * & ns & ns \\
\hline \multirow{4}{*}{$\begin{array}{l}\text { Correlation between } \\
\text { Creatinine and Electrolytes }\end{array}$} & Spearman $r$ & 0.213 & 0.385 & -0.0599 & 0.043 \\
\hline & $95 \%$ confidence interval & $\begin{array}{l}-0.140 \text { to } \\
0.518\end{array}$ & $\begin{array}{l}0.0489 \text { to } \\
0.643\end{array}$ & $\begin{array}{l}-0.409 \text { to } \\
0.305\end{array}$ & -0.304 to 0.380 \\
\hline & $P$ value (two-tailed) & 0.2194 & 0.0225 & 0.7446 & 0.806 \\
\hline & $P$ value summary & ns & * & ns & ns \\
\hline \multirow{4}{*}{$\begin{array}{l}\text { Correlation between Urea } \\
\text { and Electrolytes }\end{array}$} & Spearman $r$ & -0.215 & 0.425 & -0.00239 & 0.342 \\
\hline & $95 \%$ confidence interval & $\begin{array}{l}-0.519 \text { to } \\
0.137\end{array}$ & $\begin{array}{l}0.0967 \text { to } \\
0.670\end{array}$ & $\begin{array}{l}-0.360 \text { to } \\
0.356\end{array}$ & $\begin{array}{l}-0.000765 \text { to } \\
0.612\end{array}$ \\
\hline & $P$ value (two-tailed) & 0.2146 & 0.0109 & 0.9896 & 0.0445 \\
\hline & $P$ value summary & ns & * & ns & $*$ \\
\hline
\end{tabular}

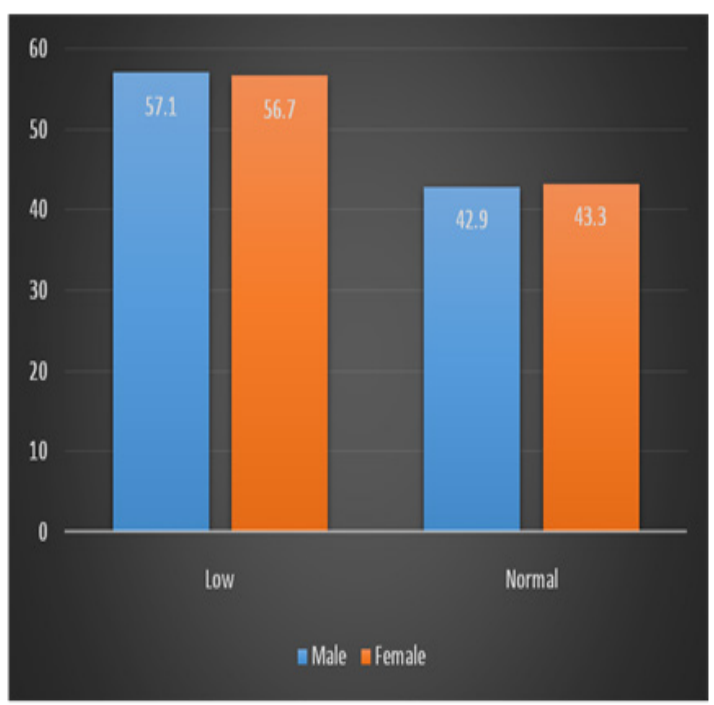

Figure 2 Distribution of patient groups according to $\mathrm{Na}+$ level.

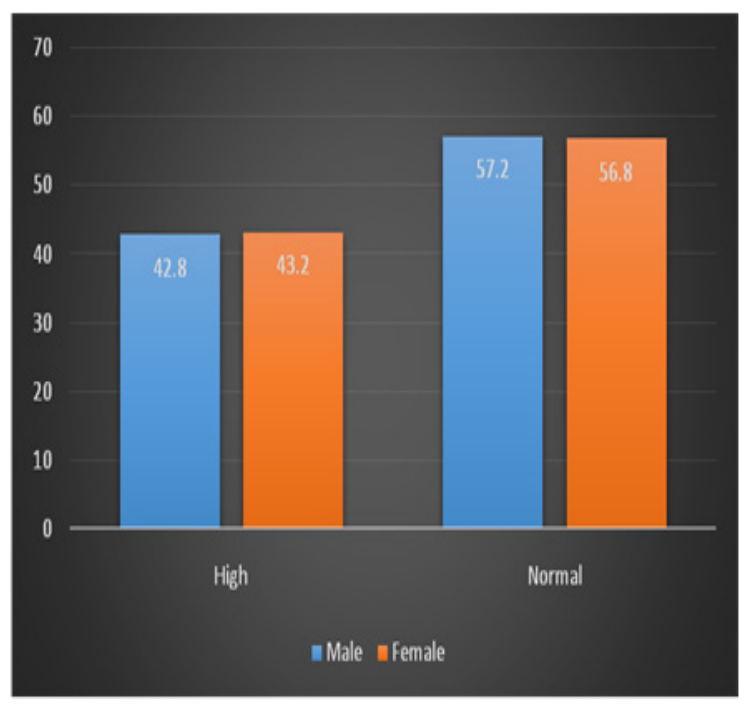

Figure 3 Distribution of patient groups according to $\mathrm{K}+$ level.

Citation: Azab AE, Elsayed ASI. Chronic kidney disease-mineral bone disease (CKD-MBD) and cardiac arrest risk factors among renal failure patients. J Appl Biotechnol Bioeng. 20 17;3(5):415-420. DOI: 10.15406/jabb.2017.03.00079 


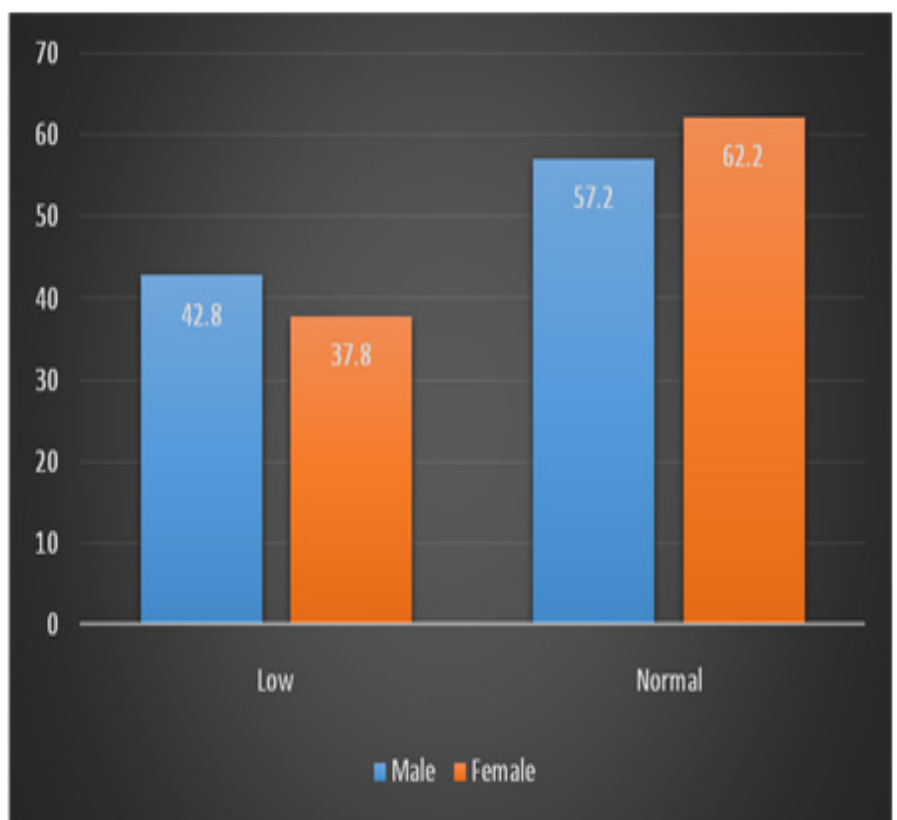

Figure 4 Distribution of patient groups according to $\mathrm{Ca}++$ levels.

Alkaline phosphatase activity was illustrated in Figure 6 and Table 1 was showed increase in means of activity in patients groups by $109 \%$ and $64 \%$ in males and females respectively with compared to control healthy persons. These data illustrated that more than $50 \%$ of patients were showed increase in the enzyme activity. The data in Figure 7 illustrated that most of patients were anemic ( $80 \%)$ and the percentage of differences between patient and control groups were $-21 \%$ and $-22 \%$ for hemoglobin in male and female patients respectively. Fifty percent of patients were diabetic, ass illustrated in Figure 8 , and the percentage of differences of means between patients and control were $68.5 \%$ for males and $73 \%$ for female patients.

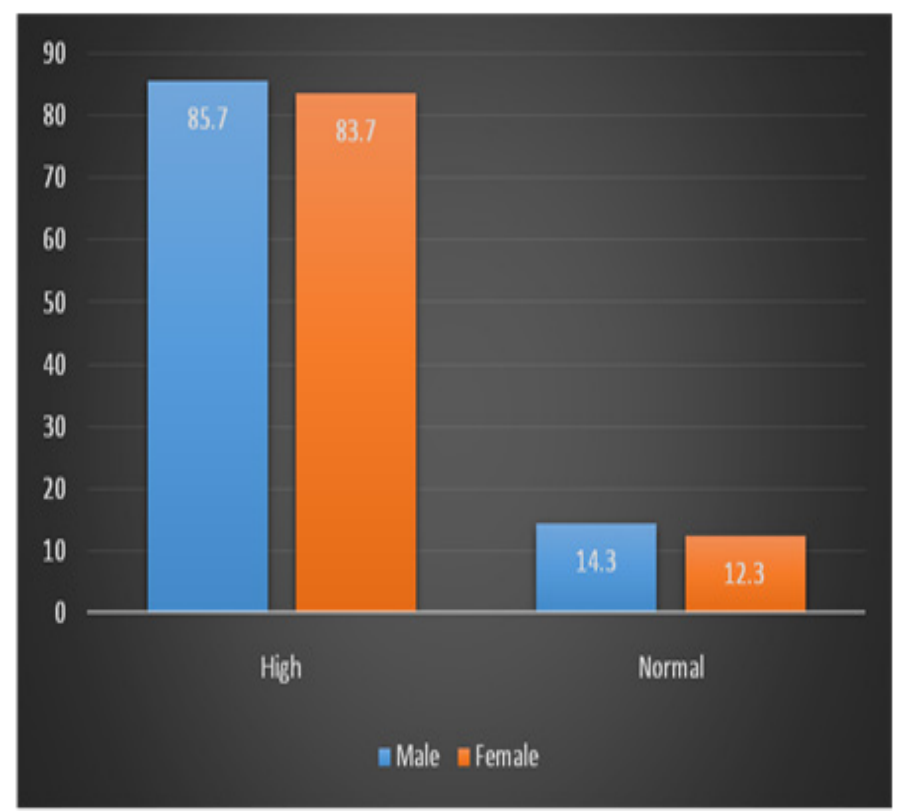

Figure 5 Distribution of patient groups according to Phosphorus level.

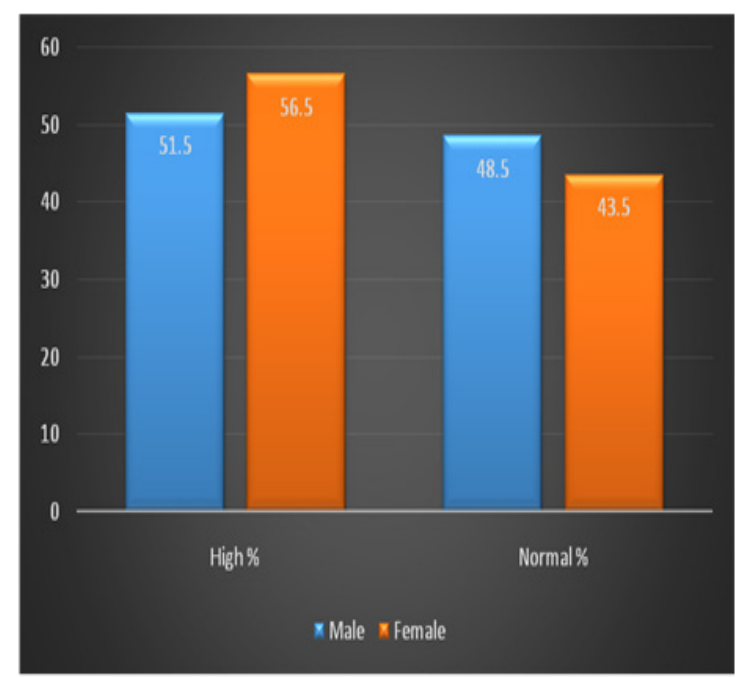

Figure 6 Distribution of patient groups according to alkaline phosphatase activity.

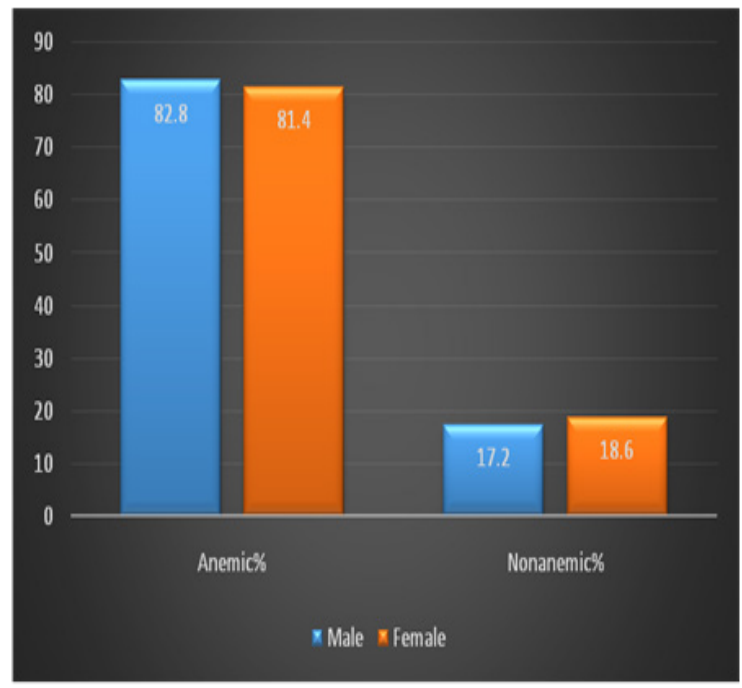

Figure 7 Distribution of patient groups according to hemoglobin concentration.

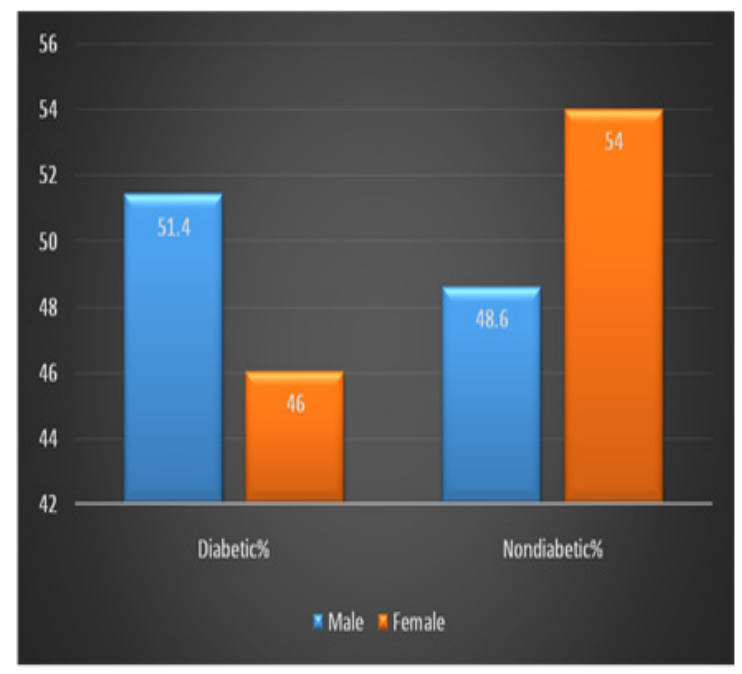

Figure 8 Distribution of patient groups according to blood sugar level. 


\section{Discussion}

Alterations in mineral bone metabolism in CKD patients are include biochemical abnormalities in calcium, phosphorous, parathyroid hormone $(\mathrm{PTH})$, vitamin $\mathrm{D}$, fibroblast growth factor-23 (FGF23 ), changes in bone volume, bone turnover, bone mineralization, calcification of soft tissue and blood vessels. ${ }^{3}$ Current understanding of CKD-MBD suggests that calcium, phosphorous, PTH, FGF-23 (a phosphatonin hormone that is elevated in CKD to promote renal phosphate excretion) and vitamin D are the key players in regulating mineral and bone metabolism. These factors are inter-related and their major target organs include parathyroid gland, kidneys, bone and intestinal tract. The classic biochemical abnormalities in CKD-MBD are hypocalcemia, hyper phosphatemia, hyper parathyroidism, hypo vitaminosis D and elevated FGF-23; however, significant variations especially in serum calcium are not uncommon. Bone abnormalities in CKD include high bone turnover disease related to secondary hyperparathyroidism (referred to as osteitis fibrosa cystica), low turnover disease (referred to as a dynamic bone disease), osteomalacia (low turnover disease accompanied by under mineralized bone tissue) and mixed disease where features of both high and low bone turnover disease are present. ${ }^{3}$ Patients with these bone abnormalities may be asymptomatic or may develop symptoms related to bone pain or fractures. Although association with bone fractures in earlier stages of CKD has been inconsistent across studies, ${ }^{9,10}$ dialysis-dependent ESRD patients have over three to four times increased risk of vertebral and hip fractures compared with general population even after adjustment for age, gender and race. Factors related to CKD-MBD including elevated PTH, vitamin D deficiency, bone remodeling, increased bone fragility and micro-structural deterioration along with increased fall risk are reported to explain this increased fracture risk in ESRD patients. As well described in the literature, CKD is characterized by low $25(\mathrm{OH})$ vitamin $\mathrm{D}$ (calcidiol), low $1,25(\mathrm{OH})_{2}$ vitamin $\mathrm{D}$ (calcitriol) as well as vitamin D resistance. ${ }^{11,12}$ Alterations related to vitamin D metabolism, Hyperphosphatemia and hypocalcemia lead to increased synthesis and/or secretion of PTH leading to secondary hyperparathyroidism that sets in as soon as GFR falls below $60 \mathrm{ml} / \mathrm{min}$. Secretion of FGF-23 from osteocytes is increased in CKD and FGF23 has been shown to reduce PTH expression via its action through Klotho-FGFR1c receptor complex. However, down regulation of this complex likely explains why despite increased FGF-23, PTH levels are not reduced in CKD. ${ }^{13}$ As early as stage 2 of CKD, serum $25(\mathrm{OH})$ vitamin $\mathrm{D}$ levels begin to decline. Reduced sun exposure, impaired skin synthesis of cholecalciferol due to renal disease, hyper pigmentation seen in late CKD stages 34 and dietary restrictions that are commonly advised to CKD patients contribute to high prevalence of vitamin D deficiency. ${ }^{14,15}$ In addition, uremia impairs intestinal absorption of dietary and supplemental vitamin $\mathrm{D}$, and in CKD patients with severe proteinuria there are high urinary losses of vitamin $\mathrm{D}$ binding protein (DBP) leading to increased renal loss of vitamin D metabolites. In addition to reasons for calcidiol deficiency in CKD patients described above, $\mathrm{CKD}$ is also marked by calcitriol deficiency. ${ }^{16,17}$

The rapid change in the extracellular concentration of electrolytes during a dialysis session leads to a secondary shift of electrolytes between the intracellular and extracellular milieu, which depends on the electrochemical gradient. as a result, cellular membrane polarization and stability may be affected. ${ }^{18}$ Hyper phosphatemia usually develops as kidney function deteriorates and $40 \%$ of patients undergoing hemodialysis has hyper phosphatemia, defined as a serum phosphate (PO4) level $>2.1 \mathrm{mmol} / 1$ (6.5mg/dl). Hyper phosphatemia provokes secondary hyperparathyroidism, smooth muscle proliferation, vascular calcification, and coronary atherosclerosis. Hyper phosphatemia-induced myocardial calcification could alter microcirculatory hemodynamics, raise extra vascular resistance and threaten myocardial perfusion. Hyperphosphatemia was associated with a mortality risk $27 \%$ higher than that of patients with Po4 levels $0.8-2.1 \mathrm{mmol} / \mathrm{l}$. Furthermore, elevated $\mathrm{Ca} \times \mathrm{Po} 4$ product $(>5.8 \mathrm{mmol} / \mathrm{l})$ was also associated with increased risk of death $(\mathrm{r}=1.34, \mathrm{P}<0.01)$. Whether this increased mortality risk is associated with an increased risk of sudden death is unknown. ${ }^{19,20}$ Divalent ion abnormalities predispose to vascular calcification of conduit vessels. In addition, these abnormalities can contribute to cardiac valve calcification and the adverse consequences of arteriosclerosis. Patients on dialysis have a two- to five fold increase in coronary artery calcification compared to the general population and this is reflected in the high prevalence of CAD. However, the vascular calcification is a diffuse phenomenon in CKD and is associated with hyper phosphataemia. There is a suggestion that phosphate and other unmeasured metabolites induce osteoblast-like activity in vascular smooth muscle. The resultant calcification means that vessels lose compliance and elasticity. Hence, there is an inability to adapt to changes in blood pressure, particularly hypotension. Vascular calcification is associated with adverse cardiovascular outcome and so may contribute to sudden cardiac death (SCD). In the presence of one or both of autonomic dysfunction and rapid fluid shift associated with dialysis, it is clear why vascular calcification may cause circulatory collapse. ${ }^{21,22}$

Cardiac autonomic neuropathy may also contribute to arrhythmia. Both diabetes and uraemia are potential causes of autonomic neuropathy. Diabetic autonomic neuropathy is in part a vascular phenomenon, but at a microvascular level. Hyperglycaemia causes neuronal vasoconstriction and oxidative stress which results in nerve ischaemia. Diabetic autonomic neuropathy is associated with potential electrocardiographic markers of risk of SCD, such as heart rate variability. However, these alone are not proven to be independent markers of risk. ${ }^{22}$ Electrolyte abnormalities are common in patients with $\mathrm{KD}^{23,24}$ Jain et al. ${ }^{5}$ reported that hyperkalemia is associated with increases in the all-cause mortality and advanced KD is an independent predictor of hyperkalemia. Hyperkalemia decreases the resting membrane potential of myocardial cells and thus reduces the conduction velocity and shortens the ventricular repolarization. These abnormalities can lead to cardiac dysrhythmias and cardiac arrests. Hyperkalemia is a reversible cause of cardiac arrest. ${ }^{25}$ Several factors render patients with KD susceptible to an imbalance of serum potassium, including chronic metabolic conditions such as diabetes mellitus, mineral corticoid deficiency, and incoherence of dietary restrictions, inadequate dialysis, hemorrhage, infections, and medications ${ }^{26} \mathrm{~A}$ slight increase in potassium intake can cause severe hyperkalemia in patients with ESRD. Medications that interfere with urinary potassium excretion or that cause a decrease in renal function may result in hyperkalemia. These medications include $\beta$-blockers, angiotensin II receptor blockers, potassium-sparing diuretics, and nonsteroid anti-inflammatory drugs. Metabolic acidosis is a common physiological disturbance during the advanced stages of chronic KD. A low serum bicarbonate may hinder the physiological mechanism to compensate acidemia. The degree of acidemia approximately correlates with the severity of KD. Poor blood perfusion in patients with cardiac arrest, even during cardiopulmonary resuscitation, also causes tissue acidosis. Acidemia increases the serum potassium 
concentration by inducing a net shift of potassium from the cellular to the extracellular compartment in exchange for hydrogen ions. ${ }^{27,28}$

\section{Conclusion}

The results of this study on chronic kidney disease patients showed that, about half of the patient groups were with hyponatremia and hyperkalemia. Calcium levels in patients was lower than control in about $40 \%$, and Phosphorus level was higher than normal in $85 \%$ of patient populations. The results illustrated that more than $50 \%$ of patients had increase in the alkaline phosphatase enzyme activity. The results also showed that most of patients were anemic (80\%) and fifty percent of patients were diabetic.

\section{Acknowledgements}

None.

\section{Conflict of interest}

The author declares no conflict of interest.

\section{References}

1. Levey AS, Stevens LA, and Coresh J. Conceptual model of CKD: Applications and implications. Am J Kidney Dis. 2009;53(3):4-16.

2. Herzog CA, Asinger RW, Berger AK, et al. Cardiovascular disease in chronic kidney disease. A clinical update from kidney disease: Improving global outcomes. Kidney Int. 2011;80:572-586.

3. Nigwekar SU, Tamez H, Thadhani RI. Vitamin D and chronic kidney disease-mineral bone disease (CKD-MBD). Bone Key Reports. 2014. p. $1-6$.

4. Bowling CB, Pitt B, Ahmed MI, et al. Hypokalemia and outcomes in patients with chronic heart failure and chronic kidney disease: Findings from propensity matched studies. Circ Heart Fail. 2010;3(2):253-260.

5. Jain N, Kotla S, Little BB, et al. Predictors of hyperkalemia and death in patients with cardiac and renal disease. Am J Cardiol. 2012;109(10):1510-1513.

6. Herzog CA. Can we prevent sudden cardiac death in dialysis patients? Clin J Am Soc Nephrol. 2007;2:410-412.

7. Bleyer AJ, Hartman J, Brannon PC, et al. Characteristics of sudden death in hemodialysis patients. Kidney Int. 2006;69(12):2268-2273.

8. Cockcroft DW, Gault MH. Prediction of creatinine clearance from serum creatinine. Nephron. 1976;16(1):31-41.

9. Ensrud KE. Fracture risk in CKD. Clin J Am Soc Nephrol. 2013;8:12821283.

10. Elliott MJ, James MT, Quinn RR, et al. Estimated GFR and fracture risk: A population-based study. Clin J Am Soc Nephrol. 2013;8(8):1367-1376.

11. Nigwekar SU, Bhan I, Thadhani R. Ergocalciferol and cholecalciferol in CKD. Am J Kidney Dis. 2012;60(1):139-156.
12. Dusso A, Gonzalez EA, Martin KJ. Vitamin D in chronic kidney disease. Best Pract Res Clin Endocrinol Metab. 2011;25:647-655.

13. Gutierrez OM. Fibroblast growth factor 23 and disordered vitamin D metabolism in chronic kidney disease: updating the "trade-off" hypothesis. Clin J AmSoc Nephrol. 2010;5(9):1710-1716.

14. Galitzer H, Ben-Dov IZ, Silver J, et al. Parathyroid cell resistance to fibroblast growth factor 23 in secondary hyperparathyroidism of chronic kidney disease. Kidney Int. 2010;77(3):211-218.

15. Del Valle E, Negri AL, Aguirre C, et al. Prevalence of $25(\mathrm{OH})$ vitamin $\mathrm{D}$ insufficiency and deficiency in chronic kidney disease stage 5 patients on hemodialysis. Hemodial Int. 2007;11(3):315-321.

16. Jacob AI, Sallman A, Santiz Z, et al. Defective photo production of cholecalciferol in normal and uremic humans. J Nutr. 1984;114(7):13131319 .

17. Abdelbaqi-Salhab M, Shalhub S, Morgan MB. A current review of the cutaneous manifestations of renal disease. J Cutan Pathol. 2003;30(9):527-538

18. Shamseddin MK, Parfrey PS. Mechanisms of the cardiorenal syndromes. Nat Rev Nephrol. 2009;5(11):641-649.

19. Schwarz U, Buzello M, Ritz E. Morphology of coronary atherosclerotic lesions in patients with end stage renal failure. Nephrol Dial Transplant. 2000;15(2):218-223.

20. Amann K, Ritz E. Microvascular disease-the Cinderella of uremic heart disease. Nephrol Dial Transplant. 2000;15(10):1493-1503.

21. De Loach SS, Townsend RR. Vascular stiffness: Its measurement and significance for epidemiologic and outcome studies. Clin J Am Soc Nephrol. 2008;3(1):184-192.

22. Roberts PR, Green D. Arrhythmias in chronic kidney disease. Heart. 2011;97(9):766-773.

23. Hsieh MF, Wu IW, Lee CC, et al. Higher serum potassium level associated with late stage chronic kidney disease. Chang Gung Med J. 2011;34(4):418-425

24. Bowling CB, Pitt B, Ahmed MI. Hypokalemia and outcomes in patients with chronic heart failure and chronic kidney disease: findings from propensity matched studies. Circ Heart Fail. 2010;3(2):253-260.

25. Neumar RW, Otto CW, Link MS. Part 8: Adult advanced cardiovascular life support: 2010 American Heart Association guidelines for cardiopulmonary resuscitation and emergency cardiovascular care. Circul. 2010;122(18):729-767.

26. Lehnhardt A, Kemper MJ. Pathogenesis, diagnosis and management of hyperkalemia. Pediatr Nephrol. 2011;26(3):377-384.

27. Kraut JA, Kurtz I. Metabolic acidosis of CKD: Diagnosis, clinical characteristics, and treatment. Am J Kidney Dis. 2005;45(6):978-993.

28. Kao KC, Huang CC, Tsai YH, et al. Hyperkalemic cardiac arrest successfully reversed by hemodialysis during cardiopulmonary resuscitation: case report. Chang Gung Med J. 2000;23(9):555-559. 